\title{
DESCRIBIENDO EL ENGAGEMENT EN PROFESIONALES DE ENFERMERÍA DE ATENCIÓN PRIMARIA DE SALUD
}

\author{
DESCRIBING THE ENGAGEMENT IN PRIMARY \\ HEALTH CARE NURSES
}

\author{
Carolina Fernández Merino* \\ Rodrigo YÁÑEZ GALLARdo **
}

\begin{abstract}
RESUMEN
En el nivel primario de atención chileno existen algunos profesionales de enfermería que, a pesar de la alta sobrecarga laboral, el difícil ambiente de trabajo y los escasos recursos asignados, continúan trabajando con gran pasión y energía. Este fenómeno se explicaría a través del constructo "Engagement". Sin embargo, a pesar de la importancia del fenómeno, éste no ha sido lo suficientemente estudiado en nuestro país, y menos en el contexto de enfermería en el nivel primario. Objetivo: describir la manifestación del Engagement en profesionales de enfermería del nivel primario de atención adscritos en la comuna de San Pedro de la Paz, Chile, año 2012. Material y método: Estudio cualitativo, descriptivo con enfoque fenomenológico y transversal, que utiliza una estrategia de estudio de casos múltiples. La muestra fue elegida a través de muestreo por intensidad, quedando finalmente compuesta por 3 enfermeras con larga trayectoria laboral, que son identificadas por las jefaturas y funcionarios de los centros de salud como profesionales con Engagement. Resultados: Se desprende de la investigación que son personas que poseen, principalmente, un gran sentido de ayuda, el cual satisfacen a través de la profesión, mostrando conductas altamente motivadas y experimentando emociones positivas muy intensas durante el contacto con los usuarios. Conclusión: Este estudio aporta importante evidencia sobre la existencia del Engagement en nuestro medio, hipotetizando que estas profesionales entienden su profesión como algo significativo, como parte de su identidad, donde satisfacen su necesidad de ayudar, y donde se valora el vínculo afectivo con los usuarios como estrategia principal.
\end{abstract}

Palabras clave: Engagement, enfermeras de salud comunitaria, recursos humanos, estudio de casos, investigación cualitativa.

\begin{abstract}
In the Chilean primary health care system, there are some nurses who, despite having a high work overload, a difficult work environment and scarce resources, continue to working with great passion and energy. This phenomenon is explained by the construct of "Engagement". However, despite the importance of this phenomenon, it has not been deeply studied in our country, especially in the context of Primary Health Care (PHC) nursing. Objective: This study aims to describe the manifestation of Engagement in nurses working in PHC centers located at the municipality of San Pedro de la Paz, Chile in 2012. Method: This is a qualitative,

\footnotetext{
* Enfermera. Alumna Programa Magíster en Psicología mención Psicología de la Salud. Universidad de Concepción. E-mail: fermerino86@gmail.com

${ }^{* *}$ Psicólogo. Docente Departamento de Psicología, Facultad de Ciencias Sociales, Universidad de Concepción, Concepción. Chile.E-mail: ryanez@udec.cl
} 
descriptive and cross-sectional study with a phenomenological approach, which uses a strategy of multiple case analyses. Sample was selected via sampling intensity. Three nurses with a long professional history, which were identified as Engagement professionals by the headquarters and staff of the health centers, were selected. Results: During the research, it was found that these nurses have, mainly, a great need to help other people, which they satisfy through their profession, showing highly motivated behaviors and experiencing intense positive emotions during the contact with patients. Conclusion: This study provides important evidence about the existence of Engagement in our environment, hypothesizing that these nurses understand their profession as meaningful, as part of their identity, and where they meet their need to help others, appreciating the affective bond with PHC users as the main strategy.

Key words: Engagement, nurses, community health, human resources, case studies, qualitative research.

Fecha recepción:13/05/13. Fecha aceptación:10/12/14.

\section{INTRODUCCIÓN}

Los centros de atención primaria en salud chilenos (APS), atienden a la gran mayoría de la población del país (1), siendo la puerta de entrada al sistema sanitario. En estos centros, los profesionales de enfermería son un pilar fundamental, por las variadas funciones que desempeñan (administrativas, asistenciales, educativas e investigativas) (2) durante todo el proceso salud-enfermedad y por todo el ciclo vital. Por esto es necesario que los profesionales de enfermería estén altamente motivados y con energía para afrontar el trabajo. Sin embargo, se ha visto que se encuentran sobreexigidos, trabajando con escasos recursos, percibiendo alto estrés laboral y sintomatología ansiosa (3).

A pesar de estas condiciones adversas, existen algunos profesionales que continúan con una alta motivación hacia el trabajo. Desde la psicología positiva ha surgido un constructo que explicaría el proceso por el cual algunas personas lograrían vivenciar una alta motivación hacia el trabajo, a pesar de las dificultades. Este constructo se llama Engagement $^{1}(4)$. Es un concepto relativa-

\footnotetext{
${ }^{1}$ En la bibliografía revisada este constructo no tiene una traducción al español. En el texto utilizaremos la palabra Engagement como sustantivo.
}

mente nuevo y que ha demostrado tener un impacto positivo en el desempeño de los profesionales de enfermería (5-9).

Pese a su importancia, se observan ciertos problemas para estudiarlo, principalmente, porque se ha adoptado de manera acrítica el constructo (10), sin lograr transculturizarlo y hacerlo pertinente a la realidad chilena, un ejemplo de esto es que no tiene una traducción al español que pueda transmitir el espíritu del constructo (11). Además, se evidencia escasa investigación del tema a nivel país, siendo aun más escasos los estudios en el nivel primario de atención. Finalmente, existen insuficientes investigaciones sobre el Engagement desde una mirada cualitativa, con prisma fenomenológico, que permitan comprender cómo se vivencia este fenómeno en los profesionales de enfermería.

\section{Comprendiendo el Engagement}

El termino Engagement fue mencionado por primera vez por Kahn en 1990 (12), quien observó que algunos trabajadores mostraban conductas altamente energéticas y motivadas en su trabajo, y esto no lograba ser explicado por constructos como compromiso institucional, liderazgo o motivación. Es así como define el Engagement como el comportamiento donde una persona utiliza y se expresa a sí misma física, cognitiva, emocional 
y mentalmente durante el desarrollo de sus roles como trabajador (11). Para este autor, una persona está con Engagement cuando se encuentra físicamente involucrada, cognitivamente vigilante y emocionalmente conectado.

Kahn (12) identifica tres condiciones psicológicas que influyen en la presencia del Engagement, las cuales son: a) Plenitud psicológica, o idea de recompensa al involucrarse de manera intensa en la labor. b) Seguridad psicológica, o seguridad de involucrarse en el trabajo sin sufrir consecuencias negativas para la autoimagen o el estatus. c) Disponibilidad psicológica, sentido de contar con los recursos personales necesarios para desempeñar favorablemente la tarea.

En los últimos años ha cobrado relevancia el estudio del Engagement, desde una nueva perspectiva, que plantea que se trataría de un estado mental positivo y satisfactorio frente al trabajo, el cual se caracteriza por tres dimensiones: vigor, dedicación y absorción (13). El vigor se refiere al alto nivel de energía y resistencia mental mientras se trabaja. El trabajo es percibido como una experiencia estimulante y energética. La dedicación implica estar totalmente involucrado en el trabajo y experimentar sentimientos de significación y entusiasmo, así como de inspiración, orgullo y reto por la tarea que se realiza. La absorción tiene que ver con tener una absoluta concentración y una fascinación con el trabajo. La persona experimenta la sensación de que el tiempo "vuela" y se deja "llevar" por lo que hace $(13,14)$.

Esta perspectiva ha sido la que ha tenido mayor relevancia científica, sobre todo luego de construirse una escala con altos índices de validez y confiabilidad, que mide las tres dimensiones antes mencionadas del Engagement, el Utrecht Work Engagement Scale (UWES) (4), el cual es el instrumento más utilizado por la literatura científica para estudiar el constructo (15).

En un estudio realizado en Chile (15), con trabajadores de sanitarios, se corrobora que el constructo se presenta en este grupo, y se observa con la aplicación del UWES la naturaleza bidimensional del Engagement, describiendo la dimensión de involucramiento o predisposición, relacionada con el grado de involucración del sujeto con la tarea, y la dimensión de satisfacción con la tarea.

Diversos estudios han revelado que el Engagement tiene una relación directa con el desempeño $(16,17)$, la predicción del clima de servicio y fidelidad al cliente (6), mejora la percepción de desempeño intra y extrarrol (7), entre otros. La relación entre el Engagement y desempeño se explicaría por cuatro mecanismos (5): La experiencia de emociones positivas que amplían el repertorio pensamiento-acción (5). El mantener un mejor estado de salud, lo que permite dedicar mayor energía a las actividades laborales (9), generación de recursos y modificación del ambiente laboral, mediante el proceso de personalización del trabajo o job crafting (7) y el contagio emocional del Engagement (18).

Investigaciones previas han revelado que los empleados con Engagement son personas optimistas y autoeficaces, capaces de manejar los eventos que afectan sus vidas. Gracias a su actitud positiva y alto nivel de actividad, estos empleados generan su propia retroalimentación positiva, en términos de apreciación, reconocimiento y éxito (19). En el contexto de enfermería se ha estudiado (20) que algunas características personales como la inteligencia emocional, resistencia y estrategias de afrontamiento son reconocidas como importantes para lidiar con las demandas del trabajo. En un grupo de enfermeras que realizan atenciones domiciliarias, se ha encontrado (20) que el sentido de autoeficacia y el optimismo (7) está altamente relacionado con el Engagement cuando las demandas emocionales experimentadas dentro de esa semana fueron altas y la presión de tiempo es más baja.

El propósito de esta investigación es describir la manifestación del fenómeno del Engagement en profesionales de enfermería 
de atención primaria de salud adscritos a la comuna de San Pedro de la Paz. Específicamente, el objetivo es describir el significado que tiene el trabajo y las manifestaciones de éste para las(los) enfermeras(os) con Engagement. Esto permitirá integrar una mirada pertinente del fenómeno en nuestra cultura, teniendo implicancias teóricas y prácticas en el campo de la enfermería.

\section{MATERIAL Y MÉTODO}

Se realizó un estudio cualitativo, descriptivo y transversal que utiliza una estrategia de estudio de casos múltiples con enfoque fenomenológico, puesto que busca conocer cómo se experimenta el Engagement desde el punto de vista de sus participantes. La estrategia utilizada fue inductiva y el diseño emergente.

Las participantes del estudio correspondieron a los profesionales de enfermería que se encontrasen trabajando en Establecimientos de Atención Primaria en Salud (CESFAM) de la comuna de San Pedro de la Paz, VIII Región, Chile, durante el año 2012, y que fuesen los más representativos de las características del Engagement según informantes claves de cada establecimiento. Esta fue una muestra teórica compuesta por el(los) profesional(es) de enfermería que mejor caracterizaran el constructo. Por tanto, se utilizó el muestreo por intensidad, ya que se buscó a los funcionarios que mejor representaran el constructo de manera intencionada. Los criterios de inclusión fueron: 1) trabajar en el establecimiento hace más de 6 meses y 2) ser reconocido como poseedor del Engagement por $2 / 3$ de los informantes consultados y 3) desempeñarse preferentemente en actividades asistenciales de atención directa con usuarios. Quedando finalmente 2 CESFAM de la comuna dentro de la muestra, y de éstos, 3 enfermeras con Engagement en total.

Las 3 enfermeras con Engagement son mujeres con más de 10 años de profesión, con características sociodemográficas y trayectos laborales comunes: a) altamente capacitadas, con estudios de especialización y/o conocimientos avanzados en áreas de salud familiar o atención usuaria (una con doble profesión), b) que eligen trabajar activamente en atención primaria, donde realizan actividades de atención directa, c) que han tenido o tienen cargos de responsabilidad en los CESFAM y que han priorizado las atenciones directas ante las labores de jefaturas o administrativas de hecho, d) han renunciado a algunos cargos de responsabilidad en pos de privilegiar el contacto con los usuarios.

Instrumento: Se elaboró una entrevista semiestructurada, la cual se aplicó a los profesionales de enfermería con Engagement. Para la construcción de ésta, previamente se entrevistó a hablantes nativos del idioma inglés, con el objetivo de comprender el significado de la palabra "Engagement" desde su idioma. De estas entrevistas se desprende que el mejor término para traducir el constructo sería la palabra "pasión". Junto con esto se complementó con bibliografía actualizada sobre el constructo, lo cual resulta en una entrevista semiestructurada de 5 temas, que busca indagar en las actividades que generan mayor motivación hacia el trabajo, intentando caracterizar las conductas, creencias y emociones ante estas labores.

Consideraciones éticas: En este estudio se resguardaron los aspectos éticos como la firma del consentimiento informado y la confidencialidad; cabe mencionar que a la fecha de realizado este estudio las instituciones participantes no contaban con comité de ética, por lo que se les solicitó la autorización por escrito para ser parte del estudio, y los criterios de rigor metodológico de credibilidad, auditabilidad y transferibilidad.

Procedimiento: Se realizó una inmersión inicial en el campo, y durante los meses de agosto a diciembre 2012 se entrevistan a los directo- 
res de los CESFAM de la comuna, con el fin de solicitar la autorización para realizar la investigación. Luego, en cada centro se entrevistan a 6 informantes claves en total (jefes de sector, enfermeras de gestión, funcionarios con larga trayectoria en el centro, entre otros), los que identifican a las 3 enfermeras con Engagement. Una vez seleccionadas(os) las profesionales, se procedió a invitarlas(os) a participar de la investigación. Las entrevistas se llevaron a cabo en las oficinas privadas de los respectivos CESFAM. Cada entrevista fue grabada por medios de audio. Estas fueron transcritas literalmente, quedando digitalizadas.

Análisis de datos: Se utilizó una bitácora de investigación. Se definió la unidad de análisis como la respuesta dada a cada pregunta de la entrevista. Se utiliza una codificación abierta y posteriormente una codificación axial para establecer las categorías. Luego de una lectura recurrente, emergen las categorías de $1^{\text {er }} y$ $2^{\circ}$ nivel. Finalmente las categorías resultantes fueron agrupadas según 3 ejes temáticos: a) percepción del trabajo, b) conducta motivada y c) emociones y sentimientos experimentados en el trabajo. Éstas fueron analizadas por análisis temático, mediante el método de comparación constante y hasta llegar al punto de saturación teórica. Las entrevistas fueron analizadas por un segundo investigador, comparando los análisis y llegando, finalmente, a las mismas conclusiones. Para el procesamiento de los datos se utilizó el programa informático $\mathrm{N}$-Vivo, de análisis y organización de los datos cualitativos.

\section{RESULTADOS}

El Engagement se manifiesta en estas enfermeras a través de los 3 ejes temáticos emergentes en las entrevistas: a) percepción en torno al trabajo, b) conducta motivada y c) emociones y sentimientos experimentados en el trabajo.

\section{a) Percepción en torno al trabajo}

Es relevante señalar que las tres enfermeras al ser preguntadas sobre lo que más les motiva sobre su trabajo, responden que es el estar directamente con los usuarios, siendo las labores asistenciales las que le dan sentido a su profesión. Es tan intenso, que las tres rechazan cargos de responsabilidad en sus centros para estar más cerca de los usuarios. Por ejemplo:

Me gusta mucho la atención directa, porque si siento que dentro de las labores de enfermería lo que más me llena a mi es la atención directa del paciente, estar en contacto con la otra persona. $Y$ eso me reconforta mucho.

Esta motivación se explica por dos razones:

i) Gran deseo de ayudar para la vida de estas mujeres. Para las tres el deseo de ayudar se manifiesta como una intención muy potente dentro del relato, incluso una refiere que es su misión de vida. El ayudar a otros las moviliza a estar atentas a los demás, intensionando acciones de ayuda, principalmente, a través de la enfermería, apoyando a sus usuarios, a quienes perciben como personas muy vulnerables, ya sea por el nivel educacional, condiciones sociales, escasas herramientas para el autocuidado, entre otras. Esta percepción las moviliza a tener una conducta de ayuda mucho más intensa. Por ejemplo:

Si tú me preguntas ahora no me podría ir de CESFAM 1, pero ¿por qué?, porque yo..., todos dicen que aquí es muy peligroso y un montón de cosas, pero sin embargo, encuentro que la gente es tan dulce, y cuando tú los tratas bien $y$ solucionas sus problemas son tan agradecidos porque ellos lo único que necesitan, y no te están pidiendo plata, ellos te están pidiendo preocupación... 
ii) Sentido de la Enfermería en sus vidas. Las enfermeras declaran que esta necesidad de ayuda la satisfacen a través de la profesión. Describen a la enfermería como "una herramienta de ayuda" a los usuarios, específicamente en ayudarlos a mejorar su calidad de vida y autocuidado. Declaran que el ser enfermeras es parte de su vida, integrando su identidad, así ellas son enfermeras todo el día y en todo momento. La profesión al mismo tiempo tiene un sentido más profundo, ligado a la espiritualidad y al proyecto de vida. Una de ellas describe la enfermería como su “apostolado". Por ejemplo:

Me gusta mi profesión, lo que hace, en donde quiera que esté yo aparte de ser yo, soy enferme$r a$, en donde sea que quiera que esté, entonces puede que yo vaya de vacaciones a una parte entonces tengo que hacer mis cosas de enfermera para ayudar a alguien que me necesite en ese momento, porque yo amo mi profesión.

\section{b) Conducta motivada}

Estas enfermeras tienen conductas en su quehacer profesional coherentes con la intensión de ayudar a los usuarios. Estas conductas las definen como profesionales con Engagement por la gran intensidad de estas acciones.

\section{i) Intensión de generar vínculos cercanos} con los usuarios. Las enfermeras reconocen una intensión de querer generar vínculos cercanos de ayuda a los usuarios. Para esto su contacto con los usuarios es más cercano y cálido, sin temor a involucrarse emocionalmente con las familias y sus historias, con una alta empatía hacia las personas y buscando generar en todo momento un ambiente físico y emocional propicio de la relación de ayuda. Por ejemplo:

Lo que más observo cuando tengo alumnos es la empatía, el poder acoger, el que el otro sienta que es un momento que le pertenece, más que a mí, a la persona que se sienta ahí (haciendo alusión donde estoy sentada). El ratito que tiene, porque le costó llegar ahí, porque no es fácil "no hay hora enfermera", y "tanto tiempo que no la veo", le dicen, bueno un ratito que trato de dedicárselo, después de entender lo que me está tratando de decir, y poderle ayudar, ... mmm... $y$ eso me hace sentir a mi bien, porque siento que esa persona se para y se va un poquito mejor, o se va con alguna reflexión también.

ii) Gran dedicación en sus labores. Las tres reconocen realizar sus labores con alto compromiso y dedicación. Por ejemplo:

Yo trato que el paciente, que las personas se den cuenta que yo estoy interesada por ellos, no sólo por lo puntual que vienen, sino que también por ellos, por sus problemas. Si yo veo que vienen con un Edimburgo alterado, por supuesto que yo no solamente voy a referirle, si no contarle qué es lo que está pasando. ¿Hay algo que tenga que contar? La escucho. No me voy a enfocar solamente a lo que está normado que tengo que hacer.

De hecho comentan realizar más de lo exigido o requerido en su trabajo para poder ayudar a los usuarios. En esto dedican tiempo y esfuerzo extra, incluso quedándose más tiempo del contratado en el CESFAM o llevándose trabajo para la casa. Por ejemplo:

Lo mismo pasa de repente con los pacientes, si me tengo que quedar aquí extra por algún paciente me quedo.

iii) Alta responsabilidad profesional. Al reconocer su labor como muy necesaria para esta población, estas enfermeras se ocupan en otorgar una atención de calidad, donde técnicamente sea lo que el usuario necesite. Para esto se están actualizando y capacitan-

\footnotetext{
${ }^{2}$ El test de Edimburgo es un instrumento de medición de depresión posparto, se le aplica a todas las madres de los lactantes de 2 y 6 meses de edad. Lo aplica el profesional de enfermería y es éste quien realiza las derivaciones en caso de que el resultado esté alterado.
} 
do constantemente, incluso una de ellas tiene una doble profesión. Esta característica está muy ligada a la gran autoexigencia que describen $2 / 3$ de las enfermeras, la cual fue una característica que identifican desde su infancia y que se propaga en todos los ámbitos de su vida. Por ejemplo:

O sea es tu ética no más la que te sostiene aquí, y que tú sabes que lo tienes que hacer bien, cada dia mejor y que lo más probable es que yo hoy día ignore cosas que yo mañana voy a saber.

\section{c) Emociones y sentimientos experimenta- dos en el trabajo}

Las tres enfermeras identifican que en su quehacer profesional perciben emociones y sentimientos muy intensos. Estas emociones se caracterizan por ser en general constantes y al describir la intensidad en una escala de autopercepción entre 1-10, creen que la intensidad de sus emociones son 8-9 de máxima intensidad. Sólo una de las tres enfermeras cree que sus emociones en el trabajo son fluctuantes y no logra definirlas de manera muy clara.

Estas emociones en general son descritas como felicidad, pasión, satisfacción y orgullo por la tarea realizada, las cuales tienen su máxima intensidad en momentos donde, al estar en relación directa con los usuarios, ellos les expresan su gratitud y confianza. También se manifiestan cuando evalúan que sus intervenciones son aceptadas por los usuarios y que tienen un efecto beneficioso en su estado de salud, mejorando sus parámetros vitales. Esto refuerza la conducta de ayuda de estas enfermeras. Por ejemplo:

...cuando hago bien mi trabajo con los pacientes siempre recibo de ellos algo que me reconforta, que es un gracias, un apretón de manos, un abrazo. Y eso me hace feliz. Sea aquí, sea en el domicilio, sea una guagüita, si es un bebé lo trato con amor, si es un adulto mayor lo trato con respeto, y de eso que yo hago siempre hay algo que me reconforta aunque a veces no me digan nada, pero sé que se van satisfechos.

\section{DISCUSIÓN Y CONCLUSIÓN}

Existen trabajadores que presentan una conducta altamente motivada en su trabajo, muestran muy alta energía, dedicación y absorción, pese a que en algunos casos puedan enfrentar condiciones laborales adversas. Esto lo llaman Engagement laboral y esta investigación ha buscado describir sus principales manifestaciones en los profesionales de enfermería que se desempeñan en un CESFAM.

A través de las entrevistas se entiende que estas profesionales son personas que valoran como parte central de sus vidas el ayudar a otros, y que satisfacen este deseo de ayudar a través de su profesión. Por esta razón se explicaría que la enfermería sea tan importante en sus vidas, ya que pasa a ser más que un trabajo, sino que su profesión es una actividad muy significativa. La enfermería, por tanto, traspasa su vida laboral y se convierte en motor motivador de sus relaciones humanas en todo ámbito y ambiente. Esto se relaciona con lo anteriormente expuesto $(12,21)$, donde las personas con Engagement muestran una alta identificación personal con la tarea.

Más específicamente, las enfermeras con Engagement, probablemente se motivan cuando se sienten útiles y valoradas, cuando piensan que pueden hacer una diferencia en la vida de los usuarios. Estos resultados refuerzan lo expuesto por Kahn (12) sobre la condición de plenitud psicológica del Engagement.

Dado lo anterior, se podría sostener que su vocación de ayuda se materializa fundamentalmente generando un vínculo cercano y una estrecha relación con los usuarios. Cabe destacar que este planteamiento no ha sido encontrado en las investigaciones revisadas, y se estima que mostrarían el rol rele- 
vante del significado del trabajo (y el potente vínculo afectivo que establecen con los usuarios) para el caso específico de enfermeras (os) con Engagement.

Intimamente relacionado con el intenso deseo de ayudar a otros, este grupo tiene en común que prefiere las labores asistenciales por sobre las administrativas o de gestión, y valoran las labores de atención directa con usuarios como las más gratificantes y significativas. A través de ellas pueden vincularse con usuarios apoyando sus distintas necesidades pero, principalmente, describen una forma de ayuda en particular, el incentivar el autocuidado en su salud en personas con problemáticas crónicas, fortaleciendo sus estrategias y empoderando a los usuarios con conductas saludables.

Esto se puede relacionar con el concepto de personalización del trabajo (22), ya que estas profesionales moldean sus tareas del trabajo en relación a sus preferencias y habilidades. Este es un aspecto muy importante en la motivación tan alta que presenta este grupo, han llegado a adecuar su puesto de trabajo haciendo lo que más les gusta.

Lo anterior también se relaciona con la dimensión de dedicación del Engagement (4, $13,16,23)$, dado que se experimenta gran sentido de significación y entusiasmo con la tarea, orgullo y reto, en este caso el ayudar a otro y el deseo de servir en sus necesidades de salud.

Como consecuencia de todo lo anterior, se ha encontrado que las enfermeras(os) con Engagement dedican siempre el máximo esfuerzo en otorgar la mejor atención posible y mejorar las estrategias para la calidad de la atención, dejando parte de sí mismas en la labor, incluso en momentos adversos. Este resultado está estrechamente relacionado a la descripción de la dimensión vigor del constructo Engagement $(11,13,16,23)$.

Todo lo anteriormente descrito nos lleva a pensar que la mejor forma de traducir la palabra Engagement en nuestro medio, sería traducirla como "pasión". Según la Real
Academia de la Lengua Española, la palabra pasión es "una inclinación o preferencia muy viva de alguien a otra persona". En este caso se hipotetiza que para estas profesionales el comprometerse en acciones de ayuda es su pasión. En ésta se entregan de manera muy energética y comprometida, experimentando emociones intensas de felicidad, disfrute, orgullo y alegría.

Por tanto, se estima que los resultados obtenidos entregan evidencia que apoya la presencia del Engagement en profesionales de enfermería de nuestro medio, y se presentaría en este grupo profesional que se dedica a las labores asistenciales de salud en contextos comunitarios (15).

Como implicaciones prácticas de esta investigación, se propone que, para desarrollar el Engagement en APS, es importante que las jefaturas propicien tareas consideradas como significativas por ellas, potenciando el sentido de ayuda hacia los usuarios. Esto se traducirá en mejores relaciones laborales y desempeño del rol.

Como limitación de esta investigación se puede señalar que se puede haber obtenido una información parcial de las entrevistadas, debido a la cantidad de participantes. Se propone en futuras investigaciones replicar la investigación en otros centros de salud familiar, para verificar si estas conclusiones se replican en otras condiciones.

Por último se sugiere hacer estudios en otras poblaciones para corroborar los resultados encontrados en esta ocasión.

\section{REFERENCIAS}

1. Fondo Nacional de Salud (FONASA). Ordinario 4H/N5924 del 14/04/2011, informa resultados del proceso de validación de población Percápita para el año 2012 [Internet]. Santiago: FONASA; 2012 [citado 14 may 2012]. 14 p. Disponible en: http://www.deis.cl/wp-content/ 
uploads/2012/01/Poblacion-inscrita-Fonasa-2012.pdf

2. Milos P, Bórquez P, Larrain A. La "gestión del cuidado" en la legislacion chilena (II). Estado actual. Cienc. enferm. 2011; XVII (3): 23-33.

3. Guic E, Mora P, Rey R, Robles A. Estrés organizacional y salud en funcionarios de centros de atencion primaria de una comuna de Santiago. Rev Med Chil. 2006; 134(1): 447-455.

4. Shaufeli W, Bakker A. UWES, Utrecht Work Engagement Scale Preliminary Manual [Internet]. The Netherlands: Occupational Health Psychology Unit, Utrecht University; 2003 [citado 12 jun 2012]. 58 p. Disponible en: http://www.beanmanaged.com/doc/pdf/arnoldbakker/articles/ articles_arnold_bakker_87.pdf

5. Palací F. Psicología de la organización. Madrid: Pearson Educación; 2005: p. 349-376.

6. Salanova M, Agut S, Peiró JM. Linking organizational resources and work engagement to employee performance and customer loyalty: The mediation of service climate. J Appl Psychol. 2005; 90(6): 1217-1227.

7. Xanthopoulou D, Bakker A, Heuven E, Demerouti E, Schaufeli W. Working in the sky: A diary study on work engagement among flight attendants. J Occup Health Psychol. 2008; 13(4): 345-356.

8. Spence H, Leiter M. The impact of nursing work environments on patient safety outcomes: the mediating role of burnout/ engagement. J Nurs Adm. 2006; 36(5): 259-267.

9. Setti I, Argentero P. Organizational features of workplace and job engagement among swiss healthcare workers. Nurs Health Sci. 2011; 13(4): 425-432.

10. Pulido-Martínez H. Produciendo trabajadores modernos: conocimiento psicológico y el mundo del trabajo en el sur. Univ. Psychol. 2007; 6(1): 27-38.

11. Silva Y, Carena M, Canuto M. Niveles de engagement y burnout en voluntarios universitarios. Un estudio exploratorio y descriptivo. Boletín de Psicología. 2013; (108): 37-57.

12. Kahn W. Psychological conditions of personal engagement and disengagement at work. Acad Manage J. 1990; 33(4): 692724.

13. Schaufeli W, Salanova M, González-Roma V, Bakker A. The measurement of engagement and burnout: A two sample confirmatory factor analytic approach. J Happiness Stud. 2002; 3(1): 71-92.

14. Bakker A, Shaufeli W, Leiter M, Taris T. Work engagement: an emerging concept in occupational health psychology. Work Stress. 2008; 22(3): 187-200.

15. Müller R, Pérez C, Ramírez L. Estructura factorial y consistencia interna de la Utrecht Work Engagement Escale (UWES) 17 entre trabajadores sanitarios de Chile. Liber. 2013; 19(2): 163-171.

16. Bakker A. An evidence-based model of work engagement. Curr Dir Psychol Sci. 2011; 20(4): 265-269.

17. Van Bogaert P, Van Heusden D, Timmermans O, Franck E. Nurse work engagement impacts job outcome and nurse-assessed quality of care: model testing with nurse practice environment and nurse work characteristics as predictors. Front Psychol. 2014; 5(1): 1-11.

18. Cruz M. El rol de la eficacia colectiva en el desempeño y bienestar de los trabajadores. Un estudio de caso en el sector de hostelería. Fòrum de Recerca. 2009; 14: 23-35.

19. Bakker A, Demerouti E. Towards a model of work engagement. Career Development International. 2008; 13(3): 209-223.

20. Bakker A, Sanz-Vergel A. Weekly work engagement and flourishing: The role of hindrance and challenge job demands. J Vocat Behav. 2013; 83(1): 397-409.

21. Simpson M. Engagement at work: a review of the literature. Int J Nurs Stud. 2009; 46(7): 1012-1024. 
22. Wrzesniewski A, Dutton J. Crafting a job: Revisioning employees as active crafters of their work. Acad Manage Rev. 2001; 26(2): 179-201
23. Bakker A, Albrecht S, Leiter M. Key questions regarding work engagement. Eur J Work Organ Psychol. 2011; 20(1): 4-28. 